Published in final edited form as:

Med Care. 2013 September ; 51(9): 812-818. doi:10.1097/MLR.0b013e31829c8ca4.

\title{
Differential receipt of sentinel lymph node biopsy within practice-based research networks
}

\author{
Anne-Marie Meyer ${ }^{1,2}$, Katherine E. Reeder-Hayes ${ }^{1,3}$, Huan Liu ${ }^{1}$, Stephanie B. Wheeler ${ }^{1,4}$, \\ Dolly Penn ${ }^{5}$, Bryan J. Weiner ${ }^{2,4}$, and William R. Carpenter ${ }^{1,2,4}$ \\ ${ }^{1}$ UNC-Lineberger Comprehensive Cancer Center, Chapel Hill, NC, USA \\ ${ }^{2}$ Cecil G. Sheps Center for Health Services Research, UNC, Chapel Hill, NC, USA \\ ${ }^{3}$ Department of Hematology/Oncology, School of Medicine, UNC, Chapel Hill, NC, USA \\ ${ }^{4}$ Department of Health Policy and Management, Gillings School of Global Public Health, \\ University of North Carolina, Chapel Hill, NC, USA \\ ${ }^{5}$ Department of Surgery, School of Medicine, UNC, Chapel Hill, NC, USA
}

\begin{abstract}
Background-Provider-based research networks (PBRNs) are promising for accelerating not only research, but also dissemination of research-based evidence into broader community practice. Sentinel lymph node biopsy (SLNB) is an innovation in breast cancer care associated with equivalent survival and lower morbidity, as compared to standard axillary lymph node dissection. We examined the diffusion of SLNB into practice and whether affiliation with the Community
\end{abstract}

Corresponding author: Anne-Marie Meyer, Lineberger Comprehensive Cancer Center, University of North Carolina at Chapel Hill, CB \#7293, Chapel Hill, NC 27599-7293, T:919.972.7468, F:919.493.8681, annemarie.meyer@unc.edu.

Author Addresses:

Anne Marie Meyer, PhD, Lineberger Comprehensive Cancer Center, CB \#7293, University of North Carolina at Chapel Hill, Chapel Hill, NC 27599-7293, T:919.972.7468, F:919.493.8681, annemarie.meyer@unc.edu

Katherine E. Reeder-Hayes, MD, MBA, University of North Carolina, Division of Hematology/Oncology, 170 Manning Dr; CB 7305, Chapel Hill, NC, USA. 27599, T: 919-966-7828, F: 919-966-6735, kreeder@med.unc.edu

Huan Liu, MS, Lineberger Comprehensive Cancer Center, University of North Carolina, 2218 W NC 54 Hwy, CB \#7293, Chapel Hill, NC 27599, T: 919-972-7477, F: 919-493-8681, huanl@email.unc.edu

Stephanie B. Wheeler, PhD, Health Policy and Management, Gillings School of Global Public Health, University of North Carolina at Chapel Hill, 1103C McGavran-Greenberg, T: 919.966.7374, F: 919.843.6362, stephanie_wheeler@unc.edu

Dolly Penn, MD, Department of Surgery, 4001 Burnett-Womack Bldg, CB \#7050, University of North Carolina at Chapel Hill, T: 919-966-4320, pennd@email.unc.edu

Bryan J. Weiner, PhD, Department of Health Policy and Management, University of North Carolina, School of Public Health, 1102A

McGavran Greenberg Hall; CB 7411, Chapel Hill, NC 27516, T: 919-966-6328, F: 919-966-6961, weiner@email.unc.edu

William R. Carpenter, PhD, Department of Health Policy and Management, University of North Carolina, School of Public Health,

1102A McGavran Greenberg Hall; CB 7411, Chapel Hill, NC 27516, T: 919-966-6328, F: 919-966-6961, bill.carpenter@unc.edu

Research Institution: University of North Carolina (Chapel Hill, NC)

Funding Disclosures:

Anne-Marie Meyer: No perceived conflicts of interest. Supported through research grant 5R01CA124402

Katherine Reeder-Hayes: No perceived conflicts of interest. Supported through research grants 5K12HD001441-12; 5 T32 HS

000032-01

Huan Liu: No perceived conflicts of interest or financial disclosures

Stephanie B. Wheeler: No perceived conflicts of interest. Supported through research grant 1-K-12 HS019468-01

Dolly Penn: No perceived conflicts of interest. Supported through research grant T32 \#5T32CA128590-04

Bryan J. Weiner: No perceived conflicts of interest. Supported through research grant 5R01CA124402

William R. Carpenter: No perceived conflicts of interest. Supported through research grant 5R01CA124402 
Clinical Oncology Program (CCOP), a cancer-focused PBRN, was associated with more rapid uptake of SLNB.

Research Design-Surveillance Epidemiology and End Results(SEER)-Medicare data were used to study women diagnosed with stage I or II breast cancer in the years 2000 to 2005 and undergoing breast conserving surgery with axillary staging $(n=6,226)$. The primary outcome was undergoing SLNB. CCOP affiliation of the surgical physician was ascertained from NCI records. Multivariable generalized linear modeling with generalized estimating equations was used to measure association between CCOP exposure and undergoing SLNB, controlling for potential confounders.

Results-Women treated by a CCOP physician had significantly higher odds of receiving SLNB compared to women treated by a non-CCOP physician (OR 2.68; 95\% CI 1.35, 5.34). The magnitude of this association was larger than that observed among patients treated by physicians operating in medical school-affiliated hospitals (OR 1.76; 95\% CI 1.30-2.39).

Conclusion-Women treated by CCOP-affiliated physicians were more likely to undergo SLNB irrespective of the hospital's medical school affiliation, suggesting that the CCOP PBRN may play a role in the rapid adoption of research-based innovation in community practice.

\section{Keywords}

Epidemiology; Breast Cancer; Practice-based research networks (PBRN); Community Clinical Oncology Program (CCOP); Sentinel Lymph Node Biopsy; SEER-Medicare

\section{Introduction}

The rapidly evolving field of cancer care demands efficient dissemination of treatment innovations. Delays in the translation of research into practice deny patients novel care that would likely produce a more favorable outcome.[1-3] One potential mechanism to accelerate the translation of cancer research into practice is through provider-based research networks (PBRNs). Embraced by the National Institutes of Health (NIH) in the Roadmap, PBRNs are research partnerships between academic investigators and community-based practitioners that expedite research by providing greater access to larger, more diverse populations. [4, 5] PBRNs are also believed to facilitate the dissemination of innovations to these populations through enhanced communication and engagement through these research partnerships.[6-8] While PBRNs are promising in this regard, there is limited empirical evidence characterizing their role in dissemination of innovative clinical practice.

To better understand the role of PBRNs in the innovation of cancer care, we examined whether diffusion of sentinel lymph node biopsy (SLNB) into practice was associated with a cancer-focused PBRN. SLNB is an innovation in the surgical approach to early stage breast cancer that has been used as an indicator of innovative breast cancer care.[9-11] Before SLNB was introduced, the more invasive axillary lymph node dissection (ALND) was the standard of care. $[9,12,13]$ Research demonstrated that SLNB is associated with similar survival benefit to ALND, yet has much less morbidity. Several health system factors have been associated with increased probability of a woman having SLNB, while at the same time there is evidence of disparities within some populations.[14, 15] 
Our study examines undergoing a SLNB procedure in the context of the National Cancer Institute's (NCI's) Community Clinical Oncology Program (CCOP), a cancer-focused PBRN that has played a substantial role in the nation's cancer clinical research.[8] Founded in 1983, this network links community-based physicians with academic investigators with the goal of improving cancer care quality in local communities through increased physician and patient participation in research and more rapid implementation of evidence-based research findings into clinical practice.[8] Physician participation in research and associated information networks have been associated with significant differences in patterns of breast cancer care.[16-18] By contrast, there is conflicting evidence regarding the association between hospital teaching status and its role in innovative breast cancer treatment.[14, 18] There is also limited evidence distinguishing the roles and interactions between physician participation and hospital participation in cancer-focused PBRNs.

Accordingly, this study examined the association between CCOP affiliation and innovation adoption, and the roles of physician participation in cancer-focused PBRNs in the context of other hospital-level factors associated with teaching and research. By doing so, it contributes important evidence to a growing body of literature empirically supporting the association between the CCOP PBRN and accelerated translation of evidence-based findings into practice. It also helps clarify our understanding of the conduits for communication and mechanisms through which such translation may occur.

\section{Methods}

\section{Data}

A retrospective cohort of breast cancer patients was identified from the Surveillance Epidemiology and End Results (SEER)-Medicare linked data.[19] The SEER-Medicare program is a collaborative effort between NCI and the Centers for Medicare and Medicaid Services (CMS). The SEER data are drawn from 17 population-based cancer registries that represent approximately 28 percent of the US cancer population. The data are comprised of patient demographic information and detailed cancer information including stage, grade, and tumor receptor status. The vast majority of Americans 65 years old and older participate in Medicare (97\%), and their claims data capture their health services utilization including information on comorbid health conditions. Linked together, SEER-Medicare data have supported numerous published studies examining the relationships between diagnoses, treatments, and outcomes through an entire course of care.[20] Our study further enriched these data to include information on the CCOP-affiliation of physicians and hospitals from the NCI CCOP Program. The CCOP data were obtained directly from the NCI CCOP program annual reports. The unique physician identification number (UPIN) for participating CCOP physicians were abstracted directly from these data.

\section{Patient Selection}

The study cohort included women who were diagnosed with stage I or II breast cancer as their first and only primary tumor between 2000 and 2005 and who received breast conserving surgery. We limited the study to women receiving breast conserving surgery because there is some ambiguity and potential for greater misclassification within 
mastectomy codes with regard to lymph node procedures compared to breast conserving surgery codes. Specifically, modified radical mastectomy (MRM) includes ALND, with no separate code for the lymph node procedure. To control for comorbidities before diagnosis and capture complete treatment information, we restricted the analysis to women age 66 and older who had complete Medicare data 12 months before diagnosis until 12 months after diagnosis. Women were also excluded if they received no treatment for breast cancer from 30 days pre-diagnosis through 12 months post-diagnosis.

\section{Main outcome and exposure}

Undergoing a SLNB procedure was the primary outcome of interest. Both SLNB and ALND were identified using International Classification of Diseases version 9 (ICD-9) Procedure codes, Current Procedural Terminology (CPT) codes, or Healthcare Common Procedure Coding System (HCPCS) codes from Medicare claims within 30 days of breast conserving surgery (BCS). Patients were categorized as undergoing SLNB if their scope of regional lymph node surgery in Medicare procedure claims indicated sentinel node removal or dye injection for identification of sentinel node. Patients were categorized as undergoing primary ALND if their procedure codes indicated axillary lymphadenectomy or if none of the criteria for SLNB were met (Appendix A). Patients who had SLNB followed by ALND were categorized as having undergone SLNB.

A patient was defined as a CCOP patient by isolating the unique UPIN on the Medicare claims for breast surgery. Of the 4,525 unique UPINs associated with the patients receiving surgery in our cohort, $1.9 \%$ of them ( 84 providers serving 874 patients) were CCOPaffiliated UPINs. Patients were considered CCOP patients if the UPIN on the breast surgery claim was that of a UPIN affiliated with the NCI CCOP program during the study period. We chose this more conservative CCOP exposure definition because we hypothesized the CCOP effect would occur via the provider most influential in surgical decision making. We also performed sensitivity analyses testing other definitions of CCOP exposure, including measure of proportion (percent of all claims associated with a CCOP physician), and comprehensive binary measure (any claim within the treatment window associated with a CCOP physician).

\section{Covariates}

Covariates selection reflected person-level, physician-level, and organization-level factors believed to be associated with treatment selection informed by our previous research and other published literature. [21-24] Person-level characteristics included age at diagnosis, race, and marital status at diagnosis [25-27]. Socio-demographic information included a patient-level measure of low income status defined by an application and approval within each state of Medicaid eligibility to support health care costs for the patient (State Buy-In). [28] Also included was a measure of the educational level in the patient's home census tract measured in quartiles within each registry and combined across registries following previous methodology.[29] Tumor information included stage, grade, and estrogen receptor status. Stage was defined according to the American Joint Committee on Cancer TNM staging system $3^{\text {rd }}$ and $6^{\text {th }}$ editions (2000-2003 and 2004-2005 cases, respectively). Medicare 
claims data were used to calculate a breast cancer specific measure of comorbidities using the NCI combined comorbidity index [30].

To control for the surgical volume of physicians (e.g., high-volume surgeons), we created a study specific measure of total breast procedures per UPIN using the claims from our patient cohort and included this as a continuous variable. Hospital organization-level measures were included to examine the other mechanisms through which there may be differential innovation diffusion. Using the SEER-Medicare Hospital file we included hospital-level measures of medical school affiliation, NCI cancer center designation, and the number of beds. NCI cancer center is included as a binary variable (Yes, No) and we categorized the number of hospital beds into quartiles. Each hospital's medical school affiliation was defined using a four-level variable in the Medicare hospital file which indicates degree of hospital affiliation with a medical school. This was retained as a binary variable in the main model where all levels of affiliation with a medical school (Major, Graduate or Limited) were grouped and compared to the referent "unaffiliated." Based on a priori hypotheses and bivariate analyses, we also tested an interaction term between CCOP and medical school affiliation.

\section{Statistical Analysis}

Bivariate analyses were conducted to examine crude associations among variables and covariate independence. To calculate the odds of undergoing SLNB, we used a generalized linear mixed model with maximum likelihood estimates based on Laplace approximation, implemented by the GLIMMIX procedure (logit link function) in SAS 9.2 (SAS, 2009). This procedure uses random effects and takes into account the hierarchical clustering of patients within physicians and physicians within hospitals when calculating estimates of association between covariates. We tested the interaction terms between CCOP and medical school affiliation. The interaction term was not statistically significant; therefore, we concluded the effect of CCOP affiliation was not moderated by medical school affiliation. To demonstrate diffusion of SLNB over the study period, figures were developed showing the crude proportion of SLNB in each of the exposure groups.

\section{Results}

The study cohort comprised 17,177 women after applying all study inclusion criteria (Figure 1); the full characteristics of the study sample are described in Table 1. The vast majority of women (95\%) received care outside of CCOP-affiliated settings ("non-CCOP") based on the affiliation of the surgeon performing their nodal dissection. Of the 854 women who were CCOP patients, 770 received SLNB. Consistent with an early-stage breast cancer cohort, there was a greater proportion of stage I (67\%), well-to-moderately differentiated (70\%), and estrogen receptor positive (77\%) tumors. Women who saw a CCOP physician were more likely to receive SLNB throughout the study period (Figure 2). From 2000 to 2005 the proportion of women receiving SLNB increased to from $73 \%$ to $96 \%$ in the CCOP sample and from $54 \%$ to $86 \%$ in the non-CCOP sample.

In multivariate models controlling physician and hospital clustering, the odds of undergoing SLNB for women treated by a CCOP physician were nearly 2.7 times that of non-CCOP 
women (OR 2.68; 95\%CI 1.35, 5.34) (Table 2). Smaller, but significant increases in odds of having SLNB were also seen in women treated at a medical school-affiliated (OR 1.76; 95\%CI 1.30, 2.39). In fact, the CCOP effect remained at the same magnitude and precision in sensitivity analyses excluding these hospital variables (OR 5.10; 95\% CI 2.58, 10.10) (data not shown). An additional sensitivity analysis tested the contrasts in the estimates of a four level-variable combining CCOP and medical school affiliation which provided even more support that CCOP affiliation was independently associated with differential receipt of treatment irrespective of hospital affiliation. (Tables $3 \& 4$ )

Women with lower-stage disease and better-differentiated tumors were also more likely to receive SLNB. However, significant racial, age and income disparities were evident in the women undergoing SLNB. Women who were African American, over 80 years of age, and with lower SES (as measured by Medicaid State-buy-in) were significantly less likely to undergo SLNB (OR 0.71; 95\% CI 0.52, 0.97 and OR 0.67; 95\% CI 0.56, 0.82, and OR 0.70; $95 \%$ CI $0.55,0.89$ respectively).

\section{Discussion}

Our study found a significant association between physician CCOP-affiliation and women's likelihood of having SLNB as a part of breast cancer surgery during the early adoption of this innovative procedure. We also found that the influence of CCOP physicians appeared to be more strongly associated with a patient having SLNB than the hospital affiliation with a medical school. This finding may challenge the perception that patients must travel to an academic medical center to receive innovative cancer care. It also indicates that within surgical treatment of breast cancer the CCOP initiative may be achieving its mission of promoting adoption of treatment innovations. Previously, we have published on an association between CCOP affiliation and innovative chemotherapeutic treatment in colon cancer.[22] Together, these studies suggest that the CCOP PBRN is associated with innovative cancer care in community settings.

There are several possible reasons for this observed association. CCOP physicians may benefit from networks and communication pathways associated with research, which may be distinct from their clinical care networks. These research networks may facilitate access to cutting-edge information in both research and practice before the information is more broadly disseminated to the clinical community. Related to this, engaging in research may foster a sense of ownership and trust of the information, as well as provide a more controlled setting through which to gain experience with the innovation and become comfortable in using it.

We examined other links to research networks as well, namely, treating hospitals' affiliation with medical schools, which have been the hubs of clinical research since prior to the development of provider-based research networks. Not surprisingly, patients treated at a medical school-affiliated hospital were more likely to undergo SLNB than those treated elsewhere. This being said, the association with innovation adoption seen through CCOP affiliation was persistently stronger than that observed for medical school affiliation. Several sensitivity tests examined multiple forms of these two measures and their interaction, and 
the observed relationships were consistent and independent. This result is plausible and important for several reasons. Among them, the independence of the measures strengthens our confidence in the study approach and findings, as the CCOP program was developed to operate outside of academic medical centers; so, the two measures should be independent by programmatic design. It also suggests the existence and the relevance of multiple communication pathways for innovation diffusion, which merits further examination.

SLNB adoption in this population does not appear to be driven by clinical trial accrual alone. While we do not have data on whether patients in our sample were recruited into a SLNB clinical trial, we did have data from the NCI which indicated trial participation by CCOP location. Using these data, we were able to estimate that almost $40 \%$ of the patients were seen in CCOPs which were not participating in any of the SLNB trials. Additionally, the total number of patients in these trials was a small fraction of those in our CCOP study sample. Finally, if there were a trial-driven adoption benefit, one would expect it to attenuate after the close of the trials; however, this study yielded utilization rates that do not converge, and instead appear to plateau, with CCOPs demonstrating persistently greater use of the new procedure.

This study has several strengths. Among them, our cohort represents a large, populationbased sample of breast cancer patients in the United States. The SEER-Medicare data allow for an extended period of observation during adoption of innovative treatment for breast cancer. With the addition of the NCI CCOP information these data represent a novel opportunity to examine physician and hospital-level influences on dissemination of treatment innovations in cancer care. Building off of previous research, we control for multiple organizational determinants and other important confounders in order to isolate the associations with the main exposure. This study also includes a hierarchical design to control for patient clustering within physicans and hospitals.

Despite these many unique strengths, our study also has several limitations. Among them, it is observational and the retrospective cohort is restricted to patients with complete follow-up information. The outcome, defined through claims, may be missing detailed clinical information such as HER2 status. This missing contextual patient information may provide important context regarding physican or patient intent and decision-making. The relative number of patients exposed to CCOPs was very small, and the data do not include a patientlevel marker of clinical trial participation. However, we have attempted to address these issues through sensitivity analyses, by examining CCOP participation in the relevant SLNB trials, and by accounting for the hierarchical structure of the data. Finally, our measure of CCOP affiliation may incompletely characterize physicians in these data. We do not have a record of when the physicians join or exit the CCOP program and are unable to create timevarying CCOP exposure. Secondly, there are different levels of practice affiliation and participation within the CCOP which we are unable to control for at the physician level[31]. Moreover, arguments may be made that there may be an element of self-selection of physicians into a CCOP which would mediate rather than confound the observed association. Specifically, CCOP practices may attract and retain physicians who are more likely than others to follow the development of new medical technologies, and identify and adopt them regardless of their affiliation with the CCOP program. The measure may also be 
a marker for other physician-level factors outside of the CCOP program, such as experience or training. These are plausable, and the exploration of possible mediation by the CCOP program or physican characteristics are important next steps for this research area.

Regardless of the exact mechanism, it is clear that patients treated by CCOP-affiliated physicians were more likely to undergo this state-of-the-art therapy than patients treated elsewhere at the time the innovation was entering clinical practice. While it was beyond the scope of the current study, future research could also examine CCOP affiliation and the differential persistence of disparities associated with race, age, and wealth illuminated by this analysis. Additionally, research that includes more detailed physician and organizational level variables could help untangle and identify more specific system attributes that promote treatment innovation. Other next steps for this area would be to incorporate these additional variables while applying more sophisticated statistical models designed for causal inference.

This study represents a unique attempt to disentangle different effects of provider and hospital affiliation in the context of innovative breast cancer care using secondary data. Our approach of combining existing (SEER-Medicare) data with unique additional information on individual providers allows us to better understand the relationships between factors that affect adoption of innovative in treatments at the institution, provider, and patient-level. Understanding the relative contribution of the different health system mechanisms will help to target interventions more effectively and influence adoption of innovative cancer care. Our findings suggest that a PBRN program, such as the CCOP, may be a good model for the dissemination of innovative cancer treatments into practice settings beyond large academic institutions.

\section{Acknowledgments}

This work was supported, in part, by the National Cancer Institute at NIH 5R01CA124402 and the Integrated Cancer Information and Surveillance System (ICISS), UNC Lineberger Comprehensive Cancer Center, through the University Cancer Research Fund through the State of North Carolina. This study used the linked SEER-Medicare database. The interpretation and reporting of these data are the sole responsibility of the authors. The authors acknowledge the efforts of the Applied Research Program, NCI; the Office of Research, Development and Information, CMS; Information Management Services (IMS), Inc.; and the Surveillance, Epidemiology, and End Results (SEER) Program tumor registries in the creation of the SEER-Medicare database. The collection of the California cancer incidence data used in this study was supported by the California Department of Public Health as part of the statewide cancer reporting program mandated by California Health and Safety Code Section 103885

\section{Bibliography}

1. Institute of Medicine. Ensuring Quality Cancer Care. Washington, DC: National Academies Press; 1999.

2. Institute of Medicine. Crossing the Quality Chasm: A New Health System for the 21 st Century. Washington DC: National Academies Press; 2001.

3. Institute of Medicine. Statement on Quality of Care. Washington, DC: National Academies Press; 1998.

4. Westfall JM, Mold J, Fagnan L. Practice-based research--"Blue Highways" on the NIH roadmap. JAMA. 2007; 297(4):403-6. [PubMed: 17244837]

5. Zerhouni E. The NIH Roadmap. Science. 2003; 302:63-72. [PubMed: 14526066]

6. Lanier D. Practice-based research networks: laboratories for improving colorectal cancer screening in primary care practice. Med Care. 2008; 46(9 Suppl 1):S147-52. [PubMed: 18725827] 
7. Sales A, et al. Models, strategies, and tools. Theory in implementing evidence-based findings into health care practice. J Gen Intern Med. 2006; 21(Suppl 2):S43-9. [PubMed: 16637960]

8. Minasian LM, et al. Translating research into evidence-based practice: the National Cancer Institute Community Clinical Oncology Program. Cancer. 2010

9. Edge SB, et al. Emergence of sentinel node biopsy in breast cancer as standard-of-care in academic comprehensive cancer centers. Journal of the National Cancer Institute. 2003; 95(20):1514-1521. [PubMed: 14559873]

10. Lyman GH, et al. American Society of Clinical Oncology guideline recommendations for sentinel lymph node biopsy in early-stage breast cancer. Journal of Clinical Oncology. 2005; 23(30):77037720. [PubMed: 16157938]

11. Cote RJ, et al. Role of immunohistochemical detection of lymph-node metastases in management of breast cancer. Lancet. 1999; 354(9182):896-900. [PubMed: 10489948]

12. Vanderveen KA, et al. Diffusion of surgical techniques in early stage breast cancer: Variables related to adoption and implementation of sentinel lymph node biopsy. Annals of Surgical Oncology. 2007; 14(5):1662-1669. [PubMed: 17285395]

13. Wright FC, et al. Adoption of Surgical Innovations: Factors Influencing Use of Sentinel Lymph Node Biopsy for Breast Cancer. Surgical Innovation. 2011; 18(4):379-386. [PubMed: 21742665]

14. Carpenter WR, et al. The role of organizational affiliations and research networks in the diffusion of breast cancer treatment innovation. Med Care. 2011; 49(2):172-9. [PubMed: 21206296]

15. Reeder-Hayes KE, et al. Race and age disparities in receipt of sentinel lymph node biopsy for early-stage breast cancer. Breast Cancer Res Treat. 2011

16. McFall SL, et al. PHYSICIAN AND PRACTICE CHARACTERISTICS ASSOCIATED WITH JUDGMENTS ABOUT BREAST-CANCER-TREATMENT. Medical Care. 1994; 32(2):106-117. [PubMed: 8302103]

17. Mandelblatt JS, et al. Measuring and predicting surgeons' practice styles for breast cancer treatment in older women. Medical Care. 2001; 39(3):228-242. [PubMed: 11242318]

18. Ayanian JZ, Guadagnoli E. Variations in breast cancer treatment by patient and provider characteristics. Breast Cancer Research and Treatment. 1996; 40(1):65-74. [PubMed: 8888153]

19. Warren JL, et al. Overview of the SEER-Medicare data: content, research applications, and generalizability to the United States elderly population. Medical Care. 2002; 40(8 Suppl):IV-3-18

20. National Cancer Institute. SEER-Medicare Publications. 2011. [cited 2012; Available from: http:// healthservices.cancer.gov/seermedicare/overview/publications.html

21. Carpenter WR, et al. A framework for understanding cancer comparative effectiveness research data needs. Journal of Clinical Epidemiology. 2012; 65(11):1150-1158. [PubMed: 23017633]

22. Carpenter WR, et al. Translating Research Into Practice The Role of Provider-based Research Networks in the Diffusion of an Evidence-based Colon Cancer Treatment Innovation. Medical Care. 2012; 50(8):737-748. [PubMed: 22437624]

23. Reeder-Hayes KE, et al. Race and age disparities in receipt of sentinel lymph node biopsy for early-stage breast cancer. Breast Cancer Research and Treatment. 2011; 128(3):863-871. [PubMed: 21340480]

24. Carpenter WR, et al. The Role of Organizational Affiliations and Research Networks in the Diffusion of Breast Cancer Treatment Innovation. Medical Care. 2011; 49(2):172-179. [PubMed: 21206296]

25. Banerjee M, et al. Disentangling the effects of race on breast cancer treatment. Cancer. 2007; 110(10):2169-2177. [PubMed: 17924374]

26. Wheeler SB, et al. Predictors of timing of adjuvant chemotherapy in older women with hormone receptor-negative, stages II-III breast cancer. Breast Cancer Research and Treatment. 2012; 131(1):207-216. [PubMed: 21842244]

27. Wheeler SB, et al. Use and Timeliness of Radiation Therapy After Breast-Conserving Surgery in Low-Income Women With Early-Stage Breast Cancer. Cancer Investigation. 2012; 30(4):258 267. [PubMed: 22489864]

28. Bach PB, et al. Patient demographic and socioeconomic characteristics in the SEER-Medicare database - Applications and limitations. Medical Care. 2002; 40(8):19-25. 
29. Keating NL, et al. Racial Differences in Definitive Breast Cancer Therapy in Older Women Are They Explained by the Hospitals where Patients Undergo Surgery? Medical Care. 2009; 47(7): 765-773. [PubMed: 19536008]

30. Klabunde $\mathrm{CN}$, et al. A refined comorbidity measurement algorithm for claims-based studies of breast, prostate, colorectal, and lung cancer patients. Annals of Epidemiology. 2007; 17(8):58490. [PubMed: 17531502]

31. Minasian LM, et al. Translating Research Into Evidence-Based Practice The National Cancer Institute Community Clinical Oncology Program. Cancer. 2010; 116(19):4440-4449. [PubMed: 20572032]

\section{Appendix}

\section{Appendix A}

List of ICD and CPT codes defining outcomes

\begin{tabular}{|l|l|l|}
\hline & ICD-9-CM Procedure codes & Procedure CPT codes \\
\hline $\begin{array}{l}\text { Breast Conservation Surgery } \\
\text { (BCS) }\end{array}$ & 85.2085 .2185 .2285 .2385 .20 & \\
\hline Mastectomy & $\begin{array}{l}85.4185 .4285 .4385 .4485 .4585 .4685 .47 \\
85.4885 .49\end{array}$ & \\
\hline $\begin{array}{l}\text { Sentinel Lymph Node Biopsy } \\
\text { (SLNB) }\end{array}$ & & $\begin{array}{l}38792,78195,38500,38525, \\
38530\end{array}$ \\
\hline $\begin{array}{l}\text { Axillary Lymph Node } \\
\text { Dissection (ALND) }\end{array}$ & & $38745,19162,19302$ \\
\hline
\end{tabular}




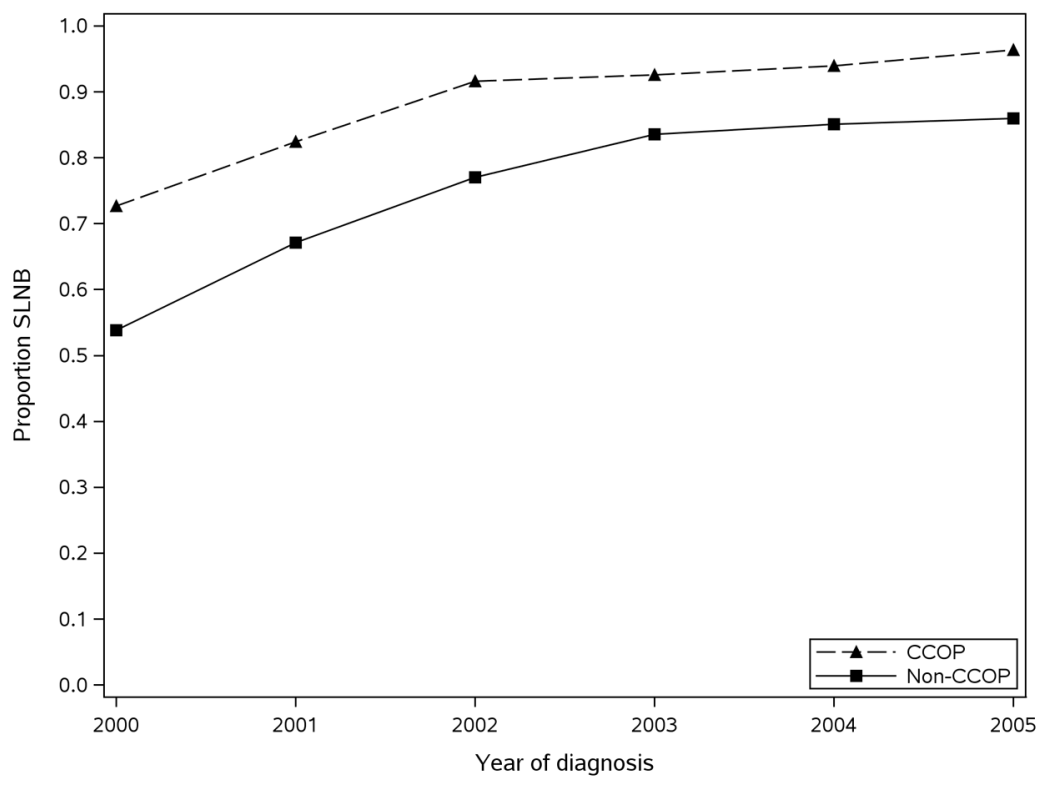

Figure 1. 


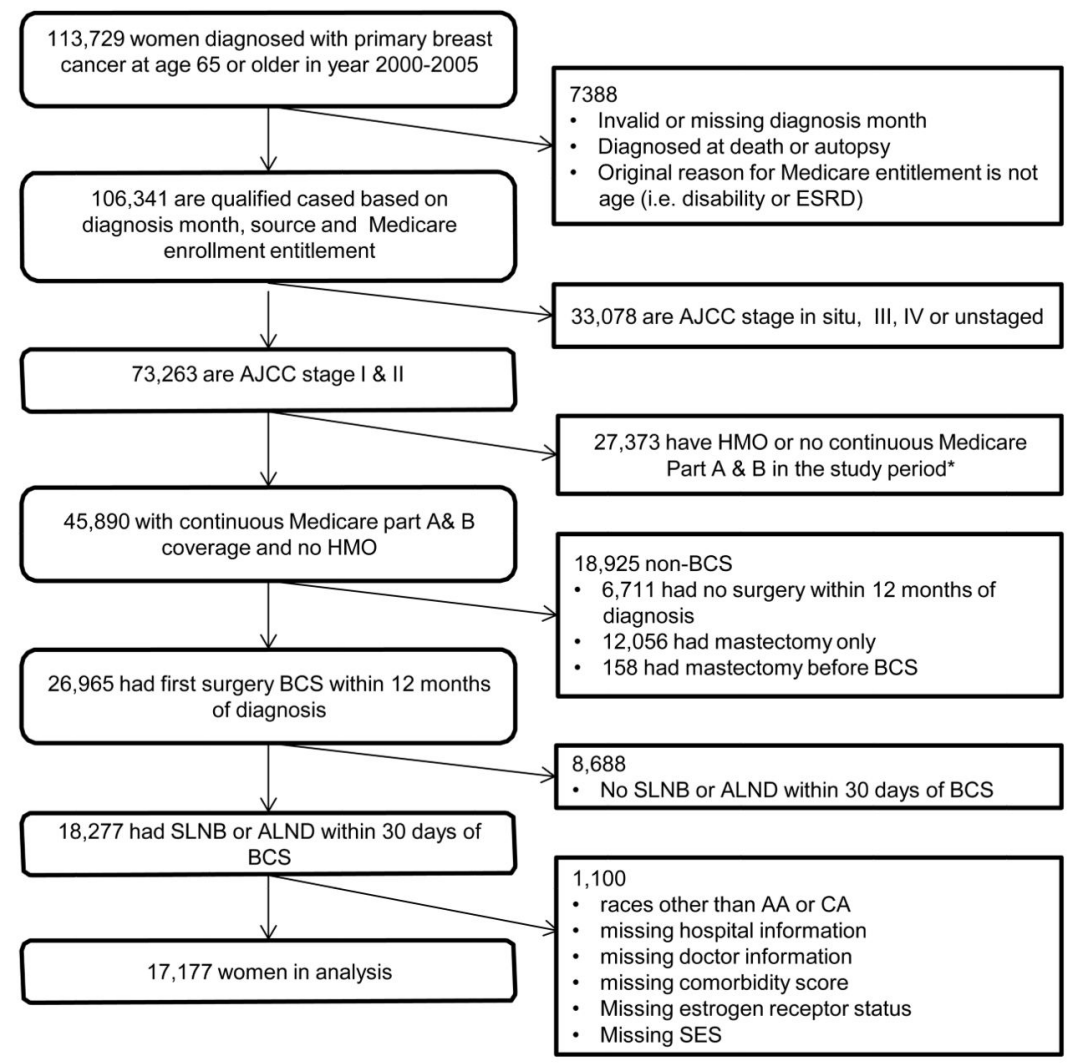

Figure 2.

* Study period is from 12 months prior to diagnosis to 12 months after diagnosis or Medicare record of death, whichever comes first. 


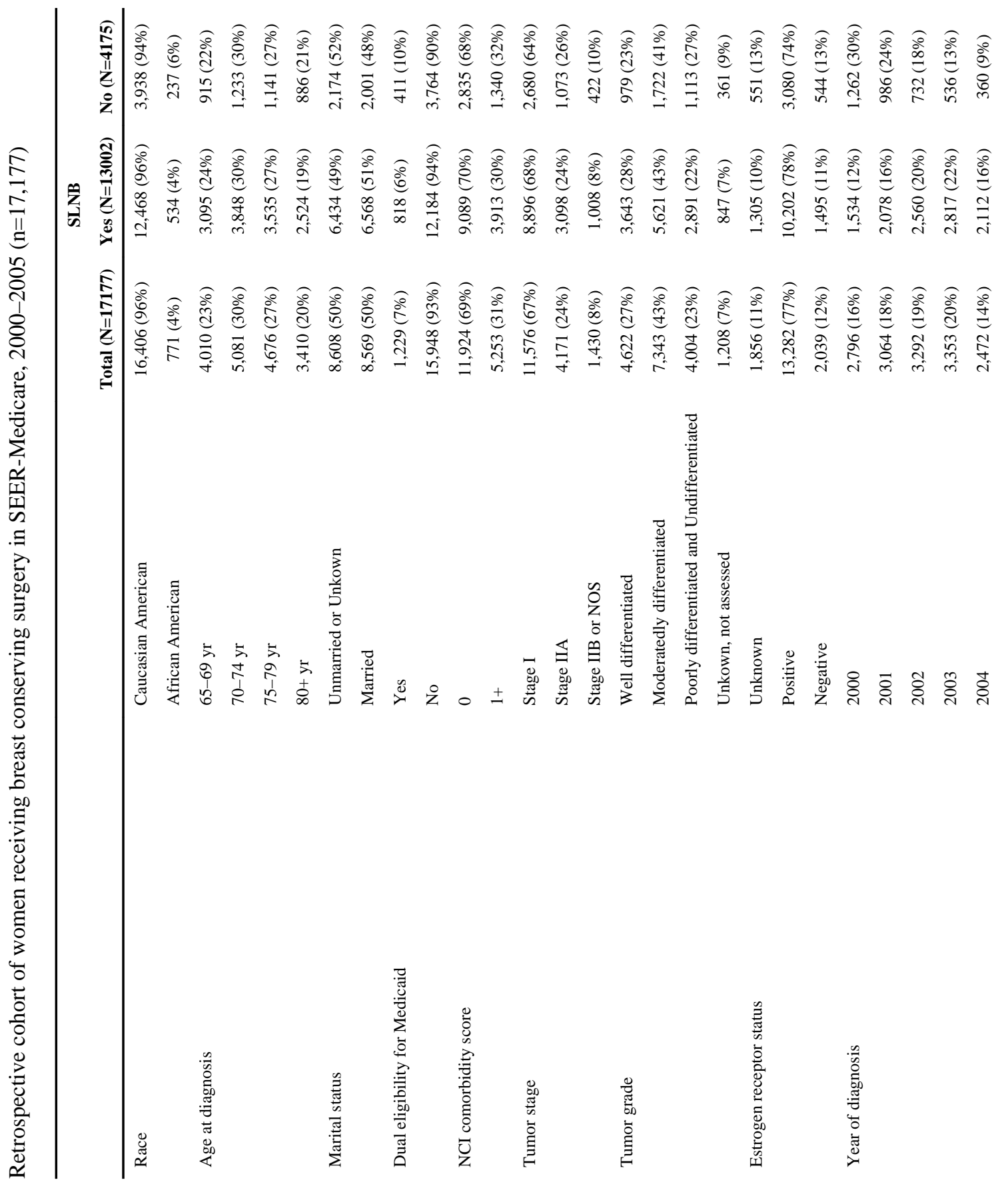




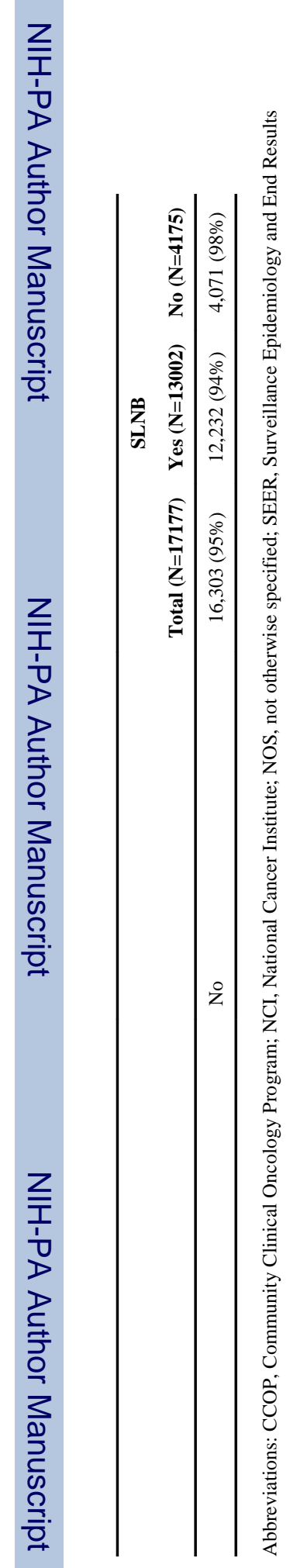


Table 2

Adjusted Odds Ratios for SLNB in women receiving breast-conserving surgery in SEER-Medicare, 2000$2005(n=17,177)$

\begin{tabular}{|c|c|c|c|}
\hline & & Adjusted Odds Ratio & $95 \% \mathrm{CI}$ \\
\hline Race (ref.= Caucasian American) & African American & $0.71^{*}$ & $(0.52-0.97)$ \\
\hline \multirow[t]{3}{*}{ Age $($ ref.= 65-69 yr) } & $70-74 \mathrm{yr}$ & 0.98 & $(0.82-1.16)$ \\
\hline & $75-79 \mathrm{yr}$ & 0.88 & $(0.74-1.06)$ \\
\hline & $80+\mathrm{yr}$ & $0.67 * *$ & $(0.56-0.82)$ \\
\hline Marital status (ref.= Married) & Unmarried or Unknown & 0.95 & $(0.83-1.09)$ \\
\hline Dual eligibility for Medicaid (ref.= No) & Yes & $0.70^{* *}$ & $(0.55-0.89)$ \\
\hline NCI comorbidity score (ref.= 1+) & 0 & 1.04 & $(0.91-1.19)$ \\
\hline \multirow[t]{2}{*}{ Tumor stage (ref.= Stage IIB or NOS) } & Stage I & $1.84 * *$ & $(1.46-2.31)$ \\
\hline & Stage IIA & $1.36^{*}$ & $(1.06-1.74)$ \\
\hline \multirow[t]{3}{*}{ Tumor grade (ref.= Poorly differentiated and Undifferentiated) } & Well differentiated & $1.57 * *$ & $(1.29-1.91)$ \\
\hline & Moderately differentiated & $1.28 * *$ & $(1.09-1.51)$ \\
\hline & Unknown, not assessed & 1.27 & $(0.98-1.64)$ \\
\hline \multirow[t]{2}{*}{ Estrogen receptor status (ref.= Positive) } & Unknown & 0.89 & $(0.72-1.11)$ \\
\hline & Negative & 0.94 & $(0.76-1.17)$ \\
\hline \multirow[t]{5}{*}{ Year of diagnosis $($ ref. $=2000$ ) } & 2001 & $2.28 * *$ & $(1.81-2.88)$ \\
\hline & 2002 & $5.22 * *$ & $(4.01-6.79)$ \\
\hline & 2003 & $10.74 * *$ & $(7.79-14.80)$ \\
\hline & 2004 & $14.01 * *$ & $(9.89-19.84)$ \\
\hline & 2005 & $13.97 * *$ & $(9.25-21.09)$ \\
\hline \multirow[t]{11}{*}{ SEER region (ref.= New Jersey) } & Connecticut & 0.91 & $(0.42-1.97)$ \\
\hline & Detroit & $0.38 *$ & $(0.19-0.76)$ \\
\hline & Hawaii & 0.53 & $(0.13-2.22)$ \\
\hline & Iowa & $0.39 *$ & $(0.19-0.80)$ \\
\hline & New Mexico & 0.74 & $(0.27-2.05)$ \\
\hline & Seattle & $5.57 * *$ & $(2.80-11.11)$ \\
\hline & Utah & 0.64 & $(0.30-1.40)$ \\
\hline & Atlanta and Rural Georgia & 1.12 & $(0.49-2.58)$ \\
\hline & All California & $0.51 *$ & $(0.31-0.83)$ \\
\hline & Kentucky & 0.99 & $(0.50-1.98)$ \\
\hline & Louisiana & 0.62 & $(0.31-1.22)$ \\
\hline \multirow{3}{*}{$\begin{array}{l}\text { Census group percent of non-high school graduates in quartiles } \\
\text { (ref.= Quartile 1) }\end{array}$} & Quartile 2: $25 \%-<50 \%$ & 0.86 & $(0.73-1.03)$ \\
\hline & Quartile 3: 50\%-<75\% & $0.81 *$ & $(0.67-0.99)$ \\
\hline & Quartile 4: > $=75 \%$ & $0.75^{* *}$ & $(0.61-0.92)$ \\
\hline \multirow[t]{3}{*}{ Hospital total bed size (ref.= Quartile 1$)$} & Quartile 2: $25 \%-<50 \%$ & 1.34 & $(0.89-2.03)$ \\
\hline & Quartile 3: 50\%-<75\% & $1.99 * *$ & $(1.26-3.16)$ \\
\hline & Quartile 4: >=75\% & $1.90^{*}$ & $(1.17-3.06)$ \\
\hline NCI cancer center designated & & $2.87 *$ & $(1.19-6.94)$ \\
\hline Medical school affiliation (Major/Grad/Limited vs. Unaffiliated) & & $1.76^{* *}$ & $(1.30-2.39)$ \\
\hline
\end{tabular}




\begin{tabular}{lrc}
\hline & Adjusted Odds Ratio & 95\% CI \\
\hline Physician surgical volume & $1.04^{* *}$ & $(1.03-1.06)$ \\
CCOP physician & $2.68^{* *}$ & $(1.35-5.34)$ \\
\hline
\end{tabular}

Abbreviations: CCOP, Community Clinical Oncology Program; CI, confidence interval; NCI, National Cancer Institute; NOS, not otherwise specified; SEER, Surveillance Epidemiology and End Results 
Table 3

Multivariate adjusted effect estimates for combined variable measuring CCOP and Medical school affiliation.

\begin{tabular}{llcc}
\hline & Exposure Levels & Adjusted Odds Ratio & 95\% CI \\
\hline $\begin{array}{l}\text { Combined Medical School Affiliation and CCOP }(\text { ref. = No } \\
\text { affiliation, non-CCOP) }\end{array}$ & (1) Major/Grad/Limited, CCOP & $3.95^{* *}$ & $(1.61-9.71)$ \\
& (2) No-affiliation, CCOP & $3.23^{* *}$ & $(1.43-7.31)$ \\
& (3) Major/Grad/Limited, non-CCOP & $1.76^{* *}$ & $(1.30-2.40)$ \\
\hline
\end{tabular}


Table 4

Contrast test for differences between effect estimates combining CCOP and Medical school affiliation.

\begin{tabular}{lccc}
\hline Contrast & Adjusted Odds Ratio & 95\% CI & Pr>ChiSq \\
\hline (1) vs (3) & 2.24 & $(0.95-5.26)$ & 0.0671 \\
(1) vs (2) & 1.22 & $(0.43-3.49)$ & 0.7056 \\
(2) vs (3) & 1.83 & $(0.80-4.18)$ & 0.1545 \\
\hline
\end{tabular}

Med Care. Author manuscript; available in PMC 2014 September 01. 